\title{
Short communication Breast screening has increased the number of mastectomies
} J Michael Dixon

Breakthrough Research Unit, Edinburgh, Edinburgh Breast Unit, Western General Hospital, Edinburgh EH4 2XU, UK

Corresponding author: J Michael Dixon, mike.dixon@ed.ac.uk

Published: 18 December 2009

This article is online at

http://breast-cancer-research.com/supplements/11/S3/S19

\section{Introduction}

The aim of breast screening is to reduce mortality and morbidity from breast cancer. One of the areas of concern in relation to breast screening is the marked increase in the number of patients with ductal carcinoma in situ (DCIS) detected through breast screening.

DCIS comprises less than $5 \%$ of all symptomatic breast cancers but now comprises more than $25 \%$ of all screendetected cancers [1]. Detecting DCIS should theoretically reduce the subsequent incidence of invasive cancer, and in terms of screening it should have the same effect as treating cervical intra-epithelial neoplasia of the cervix. DCIS has an excellent long-term outlook and there are concerns that many patients with DCIS would not have developed an invasive cancer if left untreated.

The issue in relation to DCIS therefore has to be avoiding potential overtreatment because few women die as a consequence of having DCIS, particularly if the carcinoma is low grade. Retrospective studies have found that, after excision (which often did not remove all of the DCIS), low-grade DCIS progresses to invasive cancer in only one-third of all women by 20 years [2].

\section{New data from the UK Breast Screening Programme}

The numbers of patients with DCIS has increased over the past decade. In 1998/99 there were approximately 1,500 cases, but in 2007/08 there were close to 3,500 cases (Figure 1). The percentage of patients being treated by breast-conserving surgery during this period has remained fairly constant at 30\% (Figure 2). The effect of this is that the absolute numbers of women having mastectomies has increased from just under 500 in 1998/99 to over 900 in 2007/08 (Figure 3). These data mean that the absolute numbers of patients undergoing mastectomy has almost doubled over the past decade. Although a recent statistical model estimated that women attending for screening have a
Breast Cancer Research 2009, 11(Suppl 3):S19

(doi:10.1186/bcr2438)

(c) 2009 BioMed Central Ltd

166 times higher probability of having progressive DCIS or invasive cancer than nonprogressive DCIS, there remains concern that the natural history of DCIS has not been well defined [3].

From the Sloane Project, which collects data on all patients with DCIS treated within most of the major UK centres, it is evident that some patients with DCIS are undergoing mastectomies for a radiological extent of DCIS $<40 \mathrm{~mm}$. Size is not a good predictor of the overall recurrence rate in DCIS [4]; therefore, if the DCIS can be removed adequately even if there are large areas, then mastectomy is not absolutely required even when DCIS extends beyond $4 \mathrm{~cm}$. The effectiveness of local excision is related to the fact that almost all DCIS is unicentric [4].

\section{How to address the increasing mastectomy rate for DCIS within the Screening Programme, and what are the options?}

More than $50 \%$ of DCIS lesions are oestrogen receptor rich [5]. Preoperative aromatase inhibitors reduce proliferation in DCIS [6] and tamoxifen reduces recurrence in oestrogen receptor-positive DCIS, so oestrogen receptor-positive DCIS is hormone responsive [7]. This knowledge raises the option of neoadjuvant endocrine therapy and there is some evidence that this is effective.

From studies of invasive cancer, neoadjuvant endocrine therapy reduces the overall tumour size of both the invasive component and the in situ component, producing a central scar [8]. Neoadjuvant endocrine therapy of DCIS is being further investigated in a US study [9]. Early data are promising with results from the first six patients who have completed 3 months of treatment. The three oestrogen receptor-positive DCIS patients all showed a clinical response with a decrease in tissue density rather than a reduction in extent [9]. It is clear, however, that the optimal response time to neoadjuvant hormone treatment is longer than 3 months, and 9-month or 10-month treatment durations

$\mathrm{DCIS}=$ ductal carcinoma in situ . 
Figure 1

Numbers with DCIS

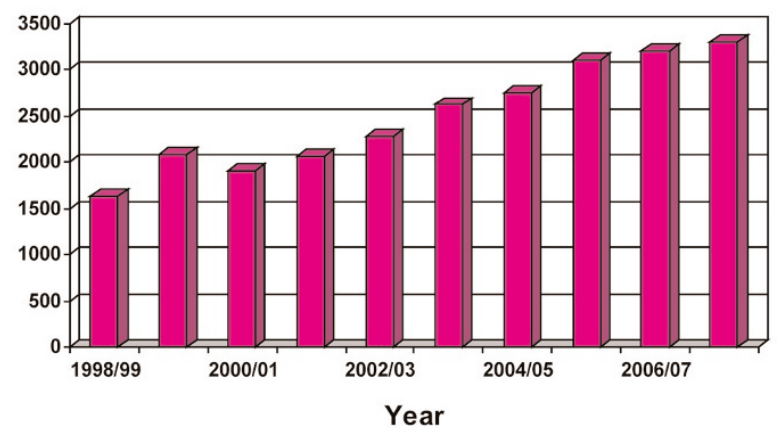

Patients diagnosed with ductal carcinoma in situ through the UK National Breast Screening Programme. Note in 1998/99 and in $1999 / 2000$ the number of patients includes ductal carcinoma in situ (DCIS) and microinvasive cancers. Data provided by Gill Lawrence and her team at the West Midlands Cancer Intelligence Unit.

\section{Figure 2}

$\%$ treated by BCS or Mastectomy

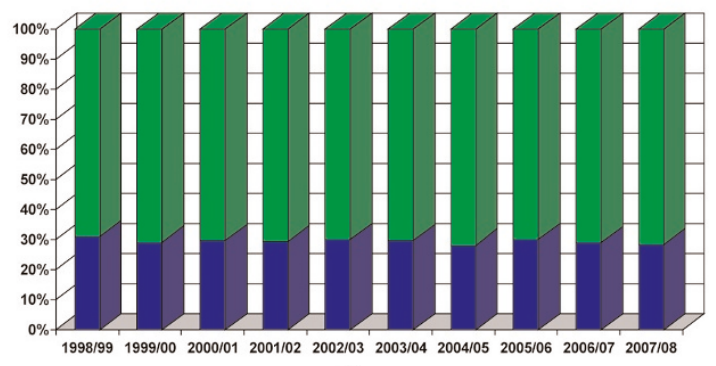

Year

Patients diagnosed with with ductal carcinoma in situ treated by mastectomy or breast-conserving surgery. Percentage of patients diagnosed with ductal carcinoma in situ (DCIS) through the UK National Breast Screening Programme treated by mastectomy or breast-conserving surgery (BCS). Note in 1998/99 and in 1999/2000 the percentage of patients includes DCIS and microinvasive cancers. Data provided by Gill Lawrence and her team at the West Midlands Cancer Intelligence Unit.

are needed in some patients with invasive cancers to achieve a meaningful reduction in tumour size [10].

Approximately $40 \%$ of DCIS overexpress HER2 [7]. Neoadjuvant trastuzumab for invasive cancer increases the pathological complete response rate from 20\% with chemotherapy alone to 40 to $50 \%$ with the same chemotherapy and trastuzumab [11]. There is evidence in the laboratory that antiHER2 therapy is effective in DCIS, and ongoing trials are evaluating whether lapatanib reduces proliferation in HER2positive DCIS [4]. Neoadjuvant anti-HER2 therapy for DCIS

\section{Figure 3}

Numbers treated by Mastectomy

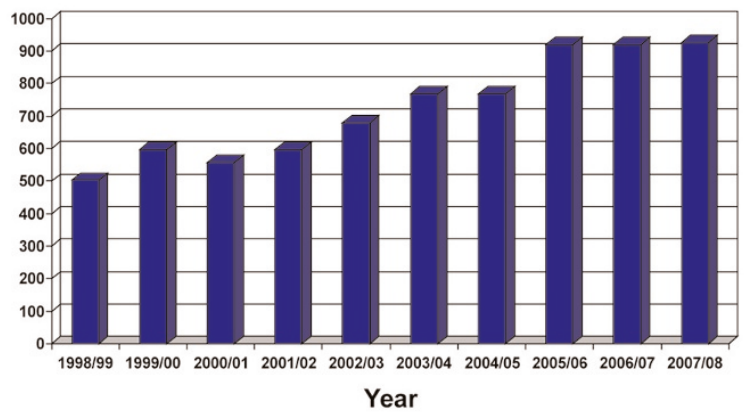

Patients diagnosed with ductal carcinoma in situ treated by mastectomy. Absolute numbers of patients diagnosed with ductal carcinoma in situ (DCIS) through the UK National Breast Screening Programme treated by mastectomy. Note in 1998/99 and in $1999 / 2000$ the number of patients includes DCIS and microinvasive cancers. Data provided by Gill Lawrence and her team at the West Midlands Cancer Intelligence Unit.

therefore requires exploring, has potential and may save some breasts.

Another option for large areas of DCIS is oncoplastic surgery, which excises the DCIS but leaves the breast shape intact and produces minimal scars. The success of breast-conserving surgery for the treatment of breast cancer is that this surgery can remove completely the local disease while preserving the natural shape and appearance of the breast [12]. Achieving the twin goals of complete tumour excision and a satisfactory cosmetic outcome can be challenging. The limiting factors are the amount of tissue that can be removed relative to the breast size and tumour location.

For larger areas of DCIS that require significant volume excision and reshaping of the affected breast, this is being combined with a simultaneous or delayed reduction mammoplasty of the opposite breast. Two surgeons, an oncoplastic surgeon and a plastic surgeon, provide the optimal approach for simultaneous bilateral mammoplasty procedures, reducing the operating time and combining the skills of both disciplines. Using oncoplastic surgery in larger breasts it is possible to excise areas of DCIS up to $10 \mathrm{~cm}$. Excising more tissue during reduction in mammoplasty approaches results in a greater incidence of clear margins, and to date the complications have been limited and the cosmetic results excellent (Figure 3). This option is not widely available but it should be the aim to offer this option for all women with larger breasts, even with extensive unifocal DCIS, who wish to be treated by breast-conserving surgery. Such women need to be fully informed of the potential advantages, risks and complications of this procedure, including incomplete excision of all disease if the disease is more extensive than 
was appreciated preoperatively or the possibility for a later mastectomy if the pathology is unfavourable.

\section{Conclusion}

It is time to try to reduce the number of mastectomies for DCIS. To achieve this reduction, more surgeons trained in oncoplastic techniques are required. There is a need for greater involvement of plastic surgeons during the discussion of treatment of such patients. Neoadjuvant endocrine and anti-HER2 therapy requires further consideration and study. The aim has to be to make the treatment fit the disease and not to make the patients fear the treatment more than the disease.

\section{Competing interests}

The author declares that they have no competing interests.

\section{Acknowledgement}

This article has been published as part of Breast Cancer Research Volume 11 Suppl 3 2009: Controversies in Breast Cancer 2009. The full contents of the supplement are available online at http://breastcancer-research.com/supplements/11/S3.

\section{References}

1. Schwartz GF, Solin LJ, Olivotto IA, Ernster VL, Pressman P; Consensus Conference Committee: The consensus conference on the treatment of in situ ductal carcinoma of the breast, 22-25 April 1999. Cancer 2000, 88:946-954.

2. Page DL, Dupont WD, Rogers LW, Jensen RA, Schuyler PA: Continued local recurrence of carcinoma 15-25 years after a diagnosis of low grade ductal carcinoma in situ of the breast treated only by biopsy. Cancer 1995, 76:1197-1200.

3. Yen MF, Tabár L, Vitak B, Smith RA, Chen HH, Duffy SW: Quantifying the potential problem of over diagnosis of ductal carcinoma in situ in breast cancer screening. Eur J Cancer 2003, 39:1746-1754.

4. Barnes NLP, Bundred NJ: Treatment of ductal carcinoma in situ. In A Companion to Specialist Surgical Practice: Breast Surgery. Edited by Dixon JM. Edinburgh: Saunders; 2009:175-192.

5. Boland GP, Knox WF, Bundred NJ: Molecular markers and therapeutic targets in ductal carcinoma in situ. Microsc Res Tech 2002, 59:3-11.

6. Dixon JM, Faratian D, White S, Renshaw L, Murray J, Young O, Macaskill EJ, Williams L, Thomas J, Evans DB: DCIS and aromatase inhibitors. J Steroid Biochem Mol Biol 2007, 106:173179.

7. Fisher B, Dignam J, Wolmark N, Wickerham DL, Fisher ER Mamounas E, Smith R, Begovic M, Dimitrov NV, Margolese RG, Kardinal CG, Kavanah MT, Fehrenbacher L, Oishi RH: Tamoxifen in the treatment of intraductal breast cancer: National Surgical Adjuvant Breast and Bowel Project B-24 randomised controlled trial. Lancet 1999, 353:1993-2000.

8. Thomas J StJ, Julian HS, Green, RV, Cameron DA, Dixon JM: Histopathology of breast carcinoma following neoadjuvant systemic therapy: a common association between letrozole therapy and central scarring. Histopathology 2007, 51:219-226.

9. Hwang ES, Esserman L: Neoadjuvant hormonal therapy for ductal carincoma in situ: trial design and preliminary results. Ann Surg Oncol 2003, 11:37S-43S.

10. Dixon JM, Renshaw L, Macaskill EJ, Young OE, Murray J, Cameron DA, Kerr GR, Evans DB, Miller WR: Increase in response rate by prolonged treatment with neoadjuvant letrozole. Breast Cancer Res Treat 2009, 113:145-151.

11. Limentani SA, Brufsky AM, Erban JK, Jahanzeb M, Lewis D: Phase II study of neoadjuvant docetexel, vinorelbine and trastuzumab followed by surgery and adjuvant doxorubicin plus cyclophosphamide in women with human epidermal growth factor receptor 2-overexpressing locally advanced breast cancer. J Clin Oncol 2007, 25:1232-1238.
12. Clough KB, Kaufman GJ, Nos C: Breast displacement techniques to increase resection volumes for breast-conserving surgery. In A Companion to Specialist Surgical Practice: Breast Surgery. Edited by Dixon JM. Edinburgh: Saunders; 2009: 86101. 